\title{
Characterization of the Eukaryotic Virome of Mice from Different Sources
}

\author{
Chunye Zhang ${ }^{1}$, Matt Burch ${ }^{1}$, Kristine Wylie ${ }^{2,3}$, Brandi Herter ${ }^{2}$, Craig L. Franklin 1,4,5,*(D) \\ and Aaron C. Ericsson 1,4,5,*iD
}

1 Department of Veterinary Pathobiology, University of Missouri, Columbia, MO 65211, USA; czvw9@mail.missouri.edu (C.Z.); mmbprd@mail.missouri.edu (M.B.)

2 Department of Pediatrics, Washington University, St. Louis, MO 63110, USA; kwylie@wustl.edu (K.W.); brandiherter@gmail.com (B.H.)

3 McDonnell Genome Institute, Washington University, St. Louis, MO 63110, USA

4 Metagenomics Center, University of Missouri, Columbia, MO 65201, USA

5 Mutant Mouse Resource and Research Center, University of Missouri, Columbia, MO 65201, USA

* Correspondence: franklinc@missouri.edu (C.L.F.); ericssona@missouri.edu (A.C.E.)

Citation: Zhang, C.; Burch, M.; Wylie, K.; Herter, B.; Franklin, C.L.; Ericsson, A.C. Characterization of the Eukaryotic Virome of Mice from Different Sources. Microorganisms 2021, 9, 2064. https://doi.org/ $10.3390 /$ microorganisms 9102064

Academic Editor: Bruce S. Seal

Received: 25 August 2021

Accepted: 29 September 2021

Published: 30 September 2021

Publisher's Note: MDPI stays neutral with regard to jurisdictional claims in published maps and institutional affiliations.

Copyright: (c) 2021 by the authors. Licensee MDPI, Basel, Switzerland. This article is an open access article distributed under the terms and conditions of the Creative Commons Attribution (CC BY) license (https:/ / creativecommons.org/licenses/by/ $4.0 /)$.

\begin{abstract}
Accumulating studies show that the host microbiome influences the development or progression of many diseases. The eukaryotic virome, as a key component of the microbiome, plays an important role in host health and disease in humans and animals, including research animals designed to model human disease. To date, the majority of research on the microbiome has focused on bacterial populations, while less attention has been paid to the viral component. Members of the eukaryotic virome interact with the commensal bacterial microbiome through transkingdom interactions, and influence host immunity and disease phenotypes as a collective microbial ecosystem. As such, differences in the virome may affect the reproducibility of animal models, and supplementation of the virome may enhance the translatability of animal models of human disease. However, there are minimal empirical data regarding differences in the virome of mice from different commercial sources. Our hypotheses were that the mice obtained from pet store sources and lab mice differ in their eukaryotic virome, and that lab mice from different sources would also have different viromes. To test this hypothesis, the ViroCap platform was used to characterize the eukaryotic virome in multiple tissues of mice from different sources including three sources of laboratory mice and two pet stores. As expected, pet store mice harbored a much greater diversity within the virome compared to lab mice. This included an ostensibly novel norovirus strain identified in one source of these mice. Viruses found in both laboratory and pet store populations included four strains of endogenous retroviruses and murine astrovirus with the latter being restricted to one source of lab mice. Considering the relatively high richness virome within different samples from healthy humans, these data suggest that mouse models from alternative sources may be more translational to the human condition. Moreover, these data demonstrate that, by characterizing the eukaryotic murine virome from different sources, novel viruses may be identified for use as field strains in biomedical research.
\end{abstract}

Keywords: gut microbiome; virome; laboratory mice; pet store mice; translatability; reproducibility; novel virus

\section{Introduction}

Animal models, especially mouse models, are used in biomedical research to investigate conditions (including chronic diseases such as autoimmune diseases, cancer, human immunodeficiency virus infection) and acute conditions (such as many infectious diseases). While the advantages of using mouse models are appreciated, there are limitations to be considered in terms of their reproducibility and accurate recapitulation of the human conditions they are used to study [1-4]. For example, one clinical study using the same drugs 
that worked very well in experimental treatment in an established mouse model of human amyotrophic lateral sclerosis (ALS) disease [5] was unable to reproduce these preclinical results when applied to a human population [3]. This example of therapeutic failure of a promising drug during clinical trials, combined with other study results [6-8] related to the reproducibility or translatability of rodent studies, suggests a need for a more thorough characterization and consideration of the mouse models used in biomedical research.

The bacterial microbiome has gained extensive attention among the biomedical research community because of its influence on many physiological parameters, and association with many adverse health outcomes. For instance, the bacterial gut microbiome is important for metabolism [9], mucosal barrier function [10], defense against certain pathogens [11], and regulation of the immune system [12,13]. Certain compositional features of the microbiome have also been associated with specific diseases such as inflammatory bowel disease [14], colorectal cancer [15] and obesity [16], among others [17]. In contrast to research on the bacterial microbiome, there are relatively few studies focused on the viral portion of the microbiome, i.e., the virome (including prokaryotic and eukaryotic viruses), a fundamental component of the host-associated microbiome $[18,19]$. Investigations of the eukaryotic virome are hindered by the lack of an efficient technique for untargeted detection of all viral nucleic acid present within a sample, with high sensitivity and specificity.

Previous studies comparing the bacterial gut microbiota of mice from different commercial sources showed significant differences in diversity and composition between the suppliers, and dramatically increased viral pathogen loads in mice obtained from nontraditional sources of mice such as pet stores [20-22]. Therefore, in the present study, we hypothesized that pet store mice would harbor a more complex eukaryotic virome than mice from traditional sources of research mice, and that lab mice from different sources would also differ in their virome. We also hypothesized that both sources (pet store and lab) of mice may contain novel viruses.

To characterize the eukaryotic virome (including both DNA and RNA viruses) present in multiple tissues of mice from different sources, weaning age mice were purchased from three commercial suppliers of laboratory mice (Jackson, Taconic, and Envigo) and two local pet stores, and multiple tissues (ileum, perianal skin, and lung) were collected and analyzed with a robust, virus isolation-independent, probe-based targeted nucleic acid enrichment approach, ViroCap [23]. ViroCap is a targeted sequence capture panel containing specific probes that target the complete genomes of 337 viral species and enables the detection of known viruses, as well as novel viruses based on sequence similarity to viral probe sequences. Using this approach, we provided a comprehensive assessment of the virome in these tissues, and characterized the viral components in the microbiome between lab mice and pet store mice.

\section{Materials and Methods}

\subsection{Ethical Approval and Informed Consent}

All studies were conducted in accordance with the recommendations put forth in the Guide for the Care and Use of Laboratory Animals and were approved by the University of Missouri Institutional Animal Care and Use Committee.

\subsection{Animals}

C57BL/ 6 mice (JAX, 4 males and 4 females) were directly purchased from Jackson Laboratory (Sacramento, CA, USA and Bar Harbor, ME, USA), C57BL/6NHsd mice (HSD, 4 males, 4 female) from Harlan Laboratory (Harlan Sprague Dawley, Indianapolis, IN, USA) and C57BL/6NTac mice (TAC, 4 males and 4 females) from Taconic (Taconic Biosciences, Inc., La Jolla, CA, USA, IN facilities). Pet store mice were purchased from Petco pet store (PS, 2 males and 2 females) and Columbia Pet Center ( 2 males and 2 females). All mice were around 4 weeks old and were post-weaning. 


\subsection{Tissue Collection}

All mice were euthanized by carbon dioxide asphyxiation and tissues including respiratory tissue (lungs; whole pluck), dermal tissue (glabrous perianal skin), and gastrointestinal tissue (ileum) were collected and flash-frozen in liquid nitrogen and stored in cryovials at $-80^{\circ} \mathrm{C}$.

\subsection{RNA, DNA Extraction and cDNA Synthesis}

Qiagen RNeasy kit (Cat \#74104) and Qiagen DNeasy blood and tissue kit (Cat \#69506) were used for RNA isolation and DNA isolation, respectively. The Agencourt AMPure XP kit (Beckman Coulter, Brea, CA, USA) was applied for cDNA purification after cDNA synthesis. A hundred $\mu \mathrm{L}$ of cDNA was processed by adding $100 \mu \mathrm{L}$ of the AMPure XP beads. Forty $\mu \mathrm{L}$ of elution buffer was added to dilute the purified cDNA according to the manufacturer's recommendations. The quality and quantity were measured by Qubit. Only one tissue type was used at a time to prevent cross-contamination of samples.

\subsection{Primer Information}

Primer A: GTTTCCCAGTCACGATANNNNNNNNN a random primer used for cDNA synthesis in the first round and the specific primer B: GTTTCCCAGTCACGATA used for the generated template application. (Primers purchased from Integrated DNA Technologies, Coralville, IA, USA).

\subsection{Library Preparation}

For the sequence library construction preparation, every 4 samples of the same type were pooled in equal volume for a single sequencing library. For instance, 4 DNA samples from the female mice sampled at the skin were pooled together and treated as one single pooling group.

Automated dual-indexed libraries were constructed with 100-250 ng cDNA or gDNA using the KAPA HTP Library Kit (KAPA Biosystems, Wilmington, MA, USA). 250 bp length inserts were targeted by using the SciClone NGS instrument (Perkin Elmer, Waltham, MA, USA). Twenty-four cDNA libraries were pooled pre-capture generating an $18 \mu \mathrm{g}$ library pool. Twenty-four gDNA libraries were pooled pre-capture generating a $27 \mu \mathrm{g}$ library pool.

\subsection{Virome Sequencing}

Both library pools were hybridized with a custom Nimblegen probe set (Roche), termed "ViroCap", targeting a pan-virome space [23]. The concentration of each captured library pool was accurately determined through qPCR (KAPA Biosystems) to produce cluster counts appropriate for the Illumina HiSeq4000 platform. One lane of $2 \times 125$ sequence data was generated per library pool yielding an average of $4 \mathrm{~Gb}$ of data per sample. Viral sequences were identified as previously described [23]. Briefly, both nucleotide and translated sequence alignments were used to identify viral reads, and data were manually reviewed. For sequence coverage determination, we used RefCov (http:/ /gmt.genome. wustl.edu/packages / refcov / accessed on 15 December 2018). Viral genomes were assembled with IDBA-UD [24], and they were manually reviewed with Tablet [25]. SAMtools [26] was used for sequence alignment evaluation.

\subsection{Statistical Analysis}

Two-way analysis of variance (ANOVA) followed by Holm-Sidak for pairwise multiple comparisons, two factors: sex and source. $p<0.05$ was considered significant difference.

\section{Results}

\subsection{Comparison of the Eukaryotic Virome of Mice from Laboratories and Pet Stores}

A subjective review of viruses detected in each group of mice revealed two clear patterns. First, pet store (PS) mice harbored a rich virome in multiple tissues while lab mice harbored a limited diversity of eukaryotic viruses. Second, several eukaryotic retroviral 
sequences were detected in all groups. Twenty-one virus species in total were detected in samples from PS mice, including both DNA and RNA viruses belonging to ten families. Within those 21 species, there were 9 DNA and 12 RNA viruses. As expected, RNA viruses (i.e., Picornaviridae, Arteriviridae, Astroviridae, Coronaviridae, and Caliciviridae) were detected from sequenced cDNA reverse-transcribed from sample RNA. In contrast, DNA viruses (i.e., Parvoviridae, Herpesviridae, Adenoviridae, and Papillomaviridae) were detected in both DNA and RNA extracted from samples, suggesting active replication by many of these viruses. Sequences matching Retroviridae were detected in both DNA and RNA from all tissues, suggesting that the former represents proviral DNA within the host genome with some active gene transcription (Figure 1).

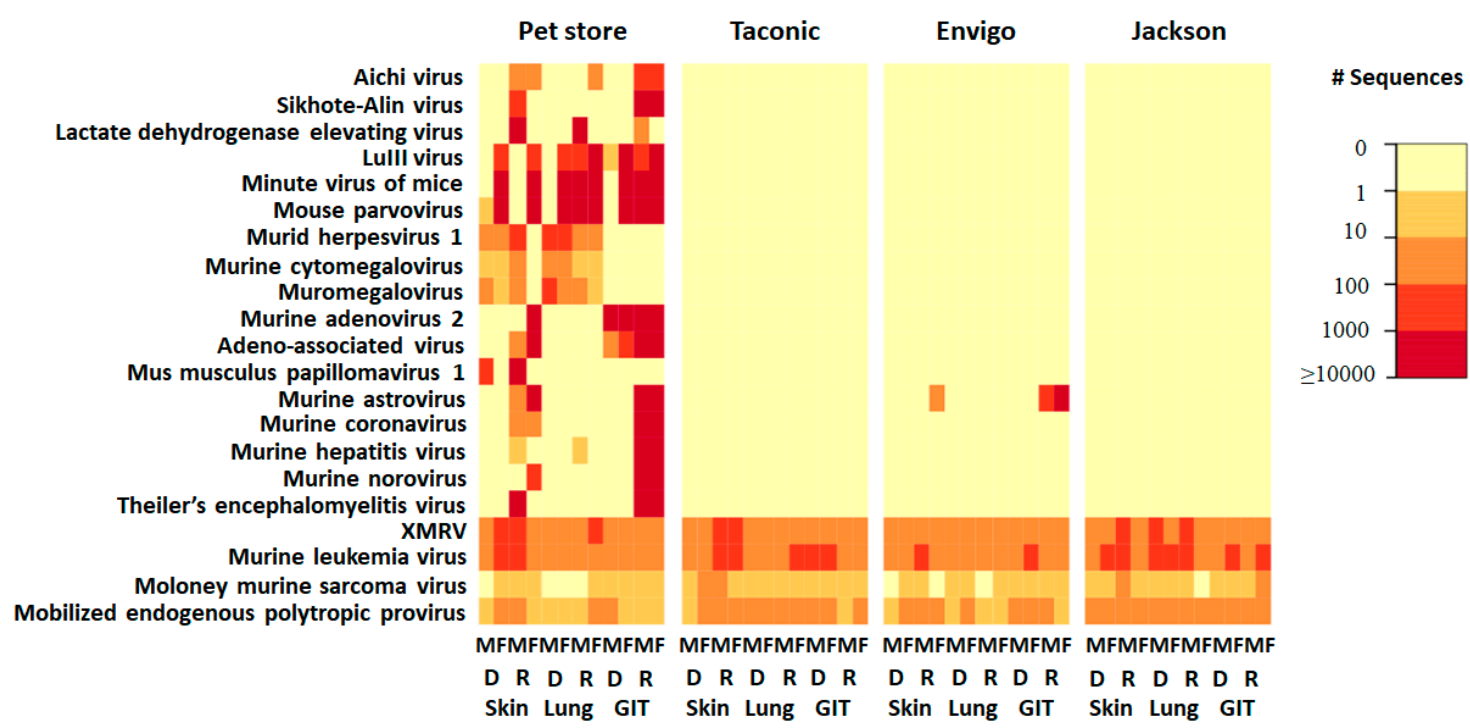

Figure 1. Heat map of vertebrate viruses from individual mice. Mice are grouped according to the source from which they were obtained: Pet Store, Taconic Farms, Envigo and the Jackson Laboratory. RNA (R) and DNA (D) were isolated from samples of gastrointestinal tissue (GIT), lung, and skin from both male (M) and female (F). Viruses detected are on the vertical axis. The value in the color key shows the range of the detected sequence number.

Aside from the Retroviridae, the remaining viruses detected in pet store mice reflected three different assigned orders, five different unassigned orders, and nine different families of viruses, ranging from Picornaviridae to Caliciviridae (Table 1).

Table 1. Categorization of viruses identified (retroviruses excluded). Table shows the summary of the identified viruses that belong to different viral families based on the common taxonomy for the classification of viruses. Besides the retrovirus family that was shared by both pet store mice and lab mice, the listed 9 viral families in this table were only found in pet store mice. * Astrovirus as the only virus found in lab mice.

\begin{tabular}{ccc}
\hline DNA/RNA Virus & Family & Virus Species \\
RNA & Picornaviridae & Aichi Virus \\
RNA & Picornaviridae & Theilers encephalomyelitis virus \\
RNA & Picornaviridae & Lactate dehydrogenase elevating virus \\
RNA & Arteriviridae & Murine astrovirus \\
RNA & Astroviridae* & Murine coronavirus \\
RNA & Coronaviridae & Murine noritis virus \\
RNA & Coronaviridae & Lull virus \\
RNA & Caliciviridae & Minute virus of mice \\
DNA & Parvoviridae & Mouse parvovirus \\
DNA & Parvoviridae & Adeno associated virus \\
DNA & Parvoviridae & Murid herpesvirus 1 \\
DNA & Parvoviridae & Murine cytomegalovirus \\
DNA & Herpesviridae & Muromegalovirus \\
DNA & Herpesviridae & Murine adenovirus 2 \\
DNA & Herpesviridae & Adenoviridae \\
DNA & Papillomaviridae & Mus musculus papillomavirus. Type 1 \\
DNA & &
\end{tabular}


Analysis of the PS mice based on sex revealed that lactate dehydrogenase elevating virus (LDEV) and mus musculus papillomavirus 1 were only detected in samples from the male mice (Figure 2). In addition, the heat map of vertebrate viruses showed there is difference between sex even among the same source such as pet store mice. Based on our knowledge, there is limited data documented on the characterization of differences on the virome between sex in lab mice.

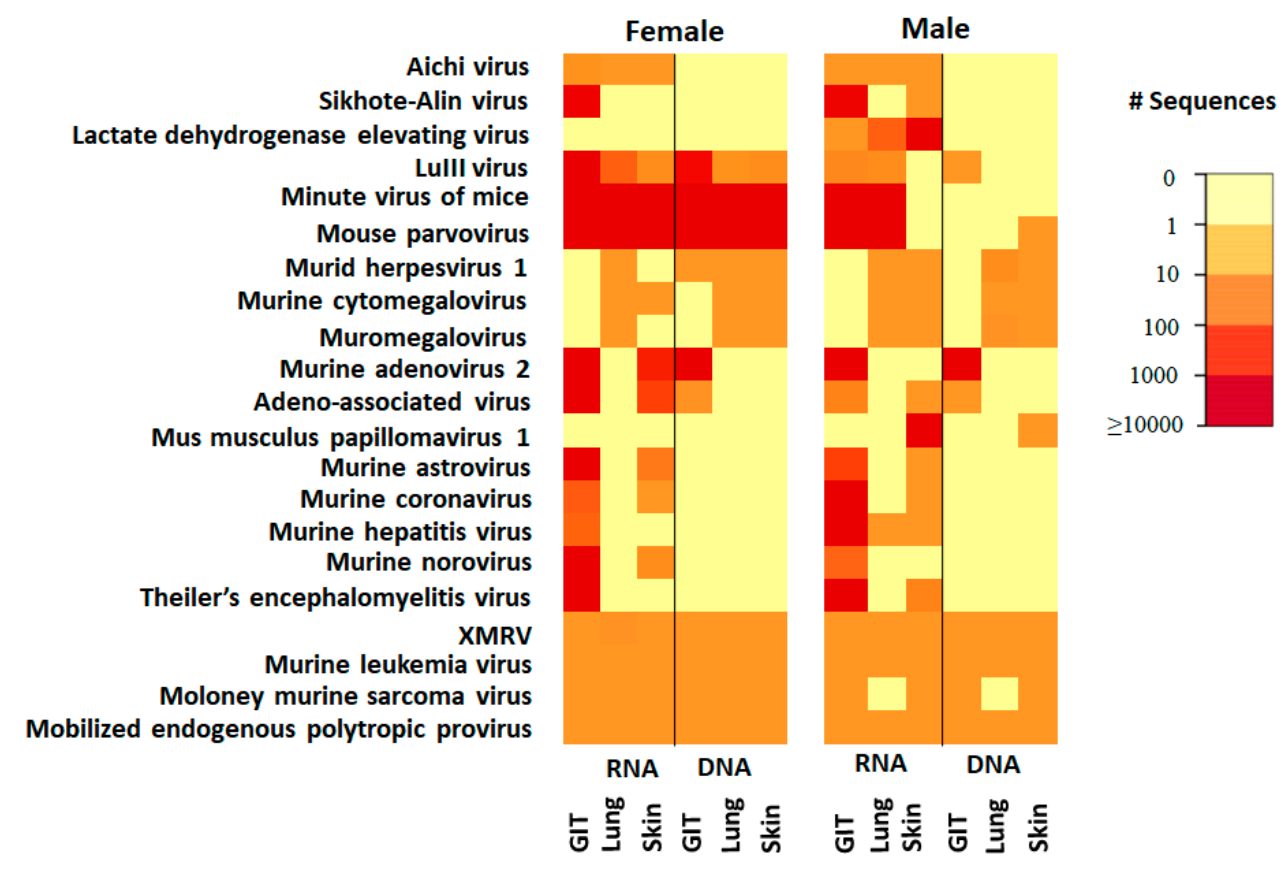

Figure 2. Heat map of vertebrate viruses from pet store mice. Mice are grouped according to the sex including female (left) and male (right): RNA (R) and DNA (D) were isolated from samples of gastrointestinal tissue (GIT), lung, and skin. Viruses detected are on the vertical axis. The value in the color key shows the range of the detected sequence number.

The number of retrovirus sequences detected differed between lab mice and PS mice. The four detected retroviruses included xenotropic murine leukemia virus-related virus (XMRV), murine leukemia virus (MLV), Moloney murine sarcoma virus, and Mus musculus mobilized endogenous polytropic provirus. Within the retrovirus, while not absolutely quantitative because the samples are pooled so not evaluated on a per-animal basis and the amplification steps in the protocol can introduce bias. Even so, the number of sequences detected suggested a trend in viral load among the retroviruses comprising greater amounts of XMRV and MLV relative to Moloney murine sarcoma and the polytropic provirus, in both lab mice and PS mice. Two-way ANOVA followed by Holm-Sidak post hoc tests detected significant differences between male JAX and male PS samples, in the number of sequences mapped to MLV $(p=0.008)$ and Moloney murine sarcoma virus $(p=0.045)$ (Figure 3).

Based on the abundance of sequences detected, the six most abundance detected viruses included mouse parvovirus, minute virus of mice, murine adenovirus 2 , murine coronavirus, murine hepatitis virus, and Theiler's encephalomyelitis virus.

\subsection{Comparison of the Eukaryotic Virome within Laboratory Mice}

Within samples from laboratory mice, one difference in the abundance of retrovirus sequences was found between mice from different vendors. Specifically, MLV was detected more frequently in samples from JAX compared to samples from $\operatorname{HSD}(p=0.037)$ (Figure 3).

Aside from the Retroviridae, astrovirus was the only other virus detected in samples in lab mice. Murine astrovirus was detected in only one of the three lab mouse suppliers (Envigo) (Figure 1). 

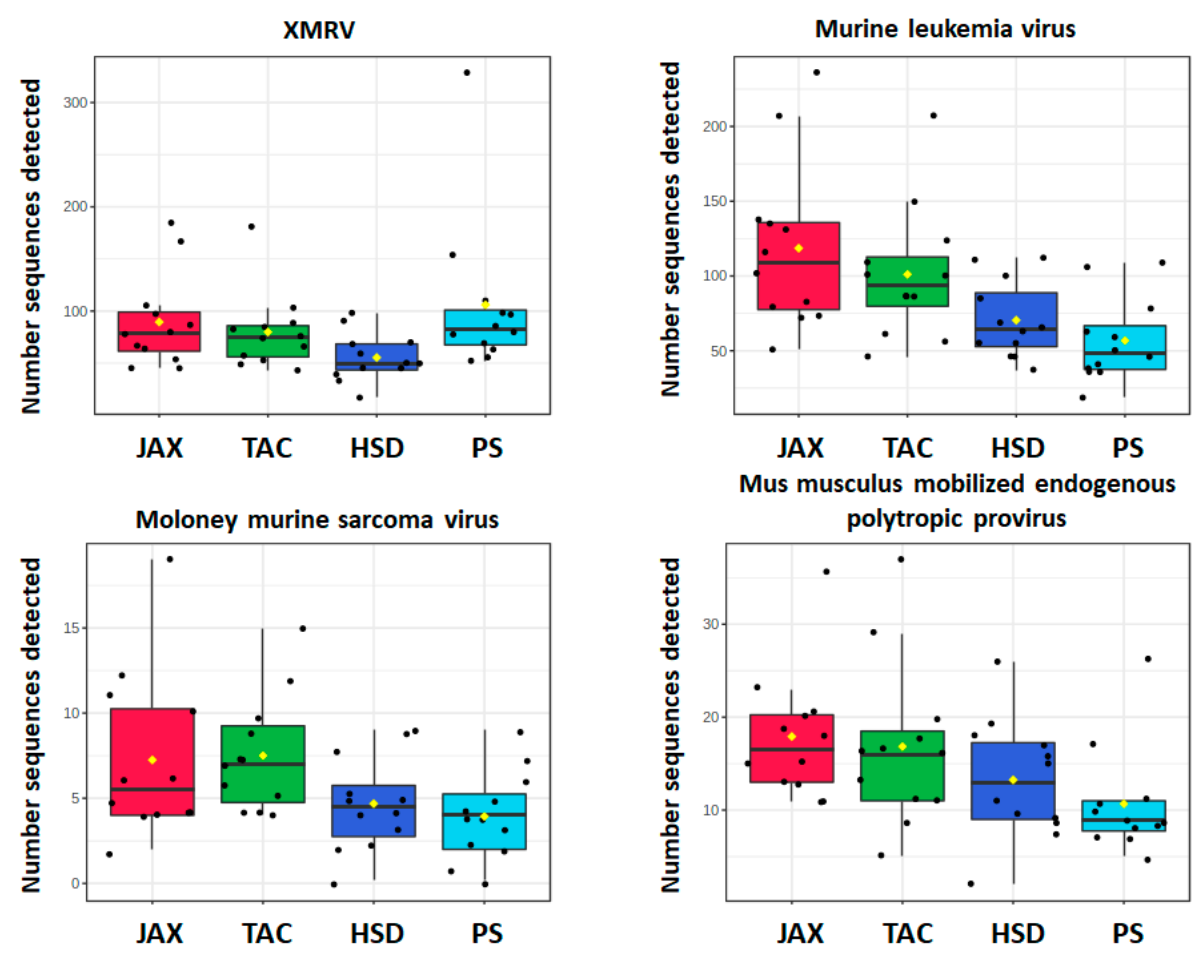

Figure 3. Number of retrovirus sequences detected in mice obtained from different sources: Jackson Laboratory (JAX), Taconic Farms (TAC), Envigo (HSD), and Pet store (PS).

\subsection{Comparison of the Viruses in Specific Tissues/Tropisms}

From each mouse, samples were collected of respiratory tissue (lungs; whole pluck), dermal tissue (glabrous perianal skin), and gastrointestinal tissue (ileum). The skin is the representative tissue tropic that exposure to the environment at large degree considering the environment is one of the sources that the host was contracted with different virus. The lung is the representative tissue for respiratory organ in the host, and the ileum is the representative tissue from the gastrointestinal which serves as reservoir for intestinal virus infection. Within the more diverse PS virome, tissue tropisms were readily apparent. The recently identified Mus musculus papillomavirus, was detected only in skin tissue (Table 2). All three members of the Herpesviridae (MHV1, MCMV, and muromegalovirus) were detected in skin and lung, but not the ileum, while murine adenovirus 2 and adenoassociated viruses were detected in the ileal and skin samples, but not the lung. Endogenous retroviruses (ERVs) were present in all tissue types.

\subsection{Potential for Novel Virus Identification}

In an effort to identify putative novel viruses, sequence of the norovirus found in PS mice from male gastrointestinal tissue sample and female gastrointestinal tissue and skin sample was compared to known murine noroviruses (Figure 4). This virus was found to share $92 \%$ sequence identity with the most-closely related strain found in GenBank. Given that noroviruses are highly mutable RNA viruses, this finding was not surprising, but reinforces that screening tools such as ViroCap can yield data on novel strains that may be worthy of further characterization and pursuit. (BioProject ID: PRJNA733600). The other viruses that were assembled did not differ greatly from previously identified/sequenced genomes. 
Table 2. Tissue specificity vertebrate viruses in pet store mice and laboratory mice.

\begin{tabular}{|c|c|c|c|c|}
\hline \multicolumn{5}{|c|}{ Tissue Specificity Vertebrate Virus in Pet Store Mice } \\
\hline Viral Family & Viral Species & GI & Lung & Skin \\
\hline Retroviridae & XMRV & $4 / 4(100 \%)$ & $4 / 4(100 \%)$ & $4 / 4(100 \%)$ \\
\hline Retroviridae & Murine leukemia viruses & $4 / 4(100 \%)$ & $4 / 4(100 \%)$ & $4 / 4(100 \%)$ \\
\hline Retroviridae & Moloney murine sarcoma virus & $1 / 4(25 \%)$ & $1 / 4(25 \%)$ & $2 / 4(50 \%)$ \\
\hline Retroviridae & Mus musculus mobilized endogenous polytropic provirus & $4 / 4(100 \%)$ & $4 / 4(100 \%)$ & $4 / 4(100 \%)$ \\
\hline Astroviridae & Murine astrovirus & $2 / 4(50 \%)$ & $0 / 4$ None & $2 / 4(50 \%)$ \\
\hline Picornaviridae & Aichi Virus & $2 / 4(50 \%)$ & $1 / 4(25 \%)$ & $2 / 4(50 \%)$ \\
\hline Picornaviridae & Skihote alin virus & $2 / 4(50 \%)$ & $0 / 4$ None & $1 / 4(25 \%)$ \\
\hline Arteriviridae & Lactate dehydrogenase elevating virus & $1 / 4(25 \%)$ & $1 / 4(25 \%)$ & $1 / 4(25 \%)$ \\
\hline Parvoviridae & LuIII virus & $3 / 4(75 \%)$ & $3 / 4(75 \%)$ & $2 / 4(50 \%)$ \\
\hline Parvoviridae & Minute virus of mice & $3 / 4(75 \%)$ & $3 / 4(75 \%)$ & $2 / 4(50 \%)$ \\
\hline Parvoviridae & Mouse parvovirus & $3 / 4(75 \%)$ & $3 / 4(75 \%)$ & $2 / 4(50 \%)$ \\
\hline Herpesviridae & Murid herpesvirus 1 & $0 / 4$ None & $4 / 4(100 \%)$ & $3 / 4(75 \%)$ \\
\hline Adenoviridae & Murine adenovirus 2 & $4 / 4(100 \%)$ & $0 / 4$ None & $1 / 4(25 \%)$ \\
\hline Parvoviridae & Adeno associated virus & $4 / 4(100 \%)$ & $0 / 4$ None & $2 / 4(50 \%)$ \\
\hline Papillomaviridae & Mus musculus papillomavirus. Type 1 & $0 / 4$ None & $0 / 4$ None & $2 / 4(50 \%)$ \\
\hline Coronaviridae & Murine coronavirus & $2 / 4(50 \%)$ & $0 / 4$ None & $2 / 4(50 \%)$ \\
\hline Coronaviridae & Murine hepatitis virus & $2 / 4(50 \%)$ & $0 / 4$ None & $1 / 4(25 \%)$ \\
\hline Caliciviridae & Murine norovirus & $2 / 4(50 \%)$ & $0 / 4$ None & $1 / 4(25 \%)$ \\
\hline Picornaviridae & Theilers encephalomyelitis virus & $2 / 4(50 \%)$ & $0 / 4$ None & $1 / 4(25 \%)$ \\
\hline \multicolumn{5}{|c|}{ Tissue Specific Vertebrate Viruses in Laboratory Mice } \\
\hline Retroviridae & XMRV & $12 / 12(100 \%)$ & $12 / 12(100 \%)$ & $12 / 12(100 \%)$ \\
\hline Retroviridae & Murine leukemia viruses & $12 / 12(100 \%)$ & $12 / 12(100 \%)$ & $12 / 12(100 \%)$ \\
\hline Retroviridae & Moloney murine sarcoma virus & $6 / 12(50 \%)$ & $8 / 12(67 \%)$ & $8 / 12(67 \%)$ \\
\hline Retroviridae & Mus musculus mobilized endogenous polytropic provirus & $12 / 12(100 \%)$ & $11 / 12(92 \%)$ & $12 / 12(100 \%)$ \\
\hline Astroviridae & Murine astrovirus & $2 / 12(17 \%)$ & $0 / 12$ None & $1 / 12(8 \%)$ \\
\hline
\end{tabular}

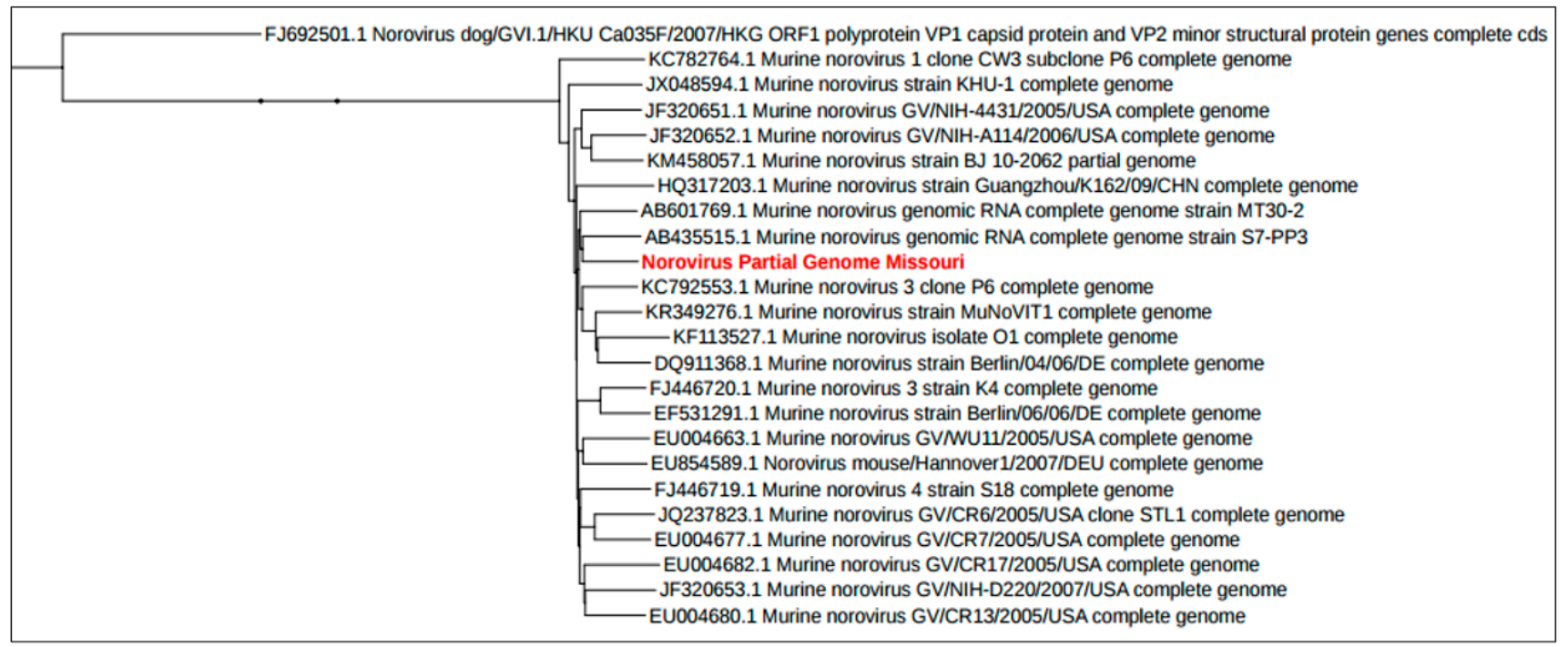

Figure 4. Phylogenetic tree of murine noroviruses. A single representative dendrogram showing the phylogenetic relationship of one potentially unique virus (shown in red) identified in pet store mice ( $92 \%$ nucleotide sequence identity to the next most-closely related strain).

\section{Discussion}

While the majority of microbiota research focuses on the bacterial component, characterization of the eukaryotic virome has lagged due to the lack of efficient methods to comprehensively survey viromes. Viruses, unlike bacteria, lack a universal conserved gene (such as the 16S rRNA gene in bacteria) enabling the identification and classification of different community members based on variable regions within that conserved gene. Virome identification, on the other hand, is complicated by the large diversity of viral genomes which do not share any universal phylogenetic marker, can be made from RNA or DNA, and can vary greatly in size and structure $[27,28]$. While shotgun metagenomic 
sequencing has been used to identify eukaryotic viral sequences, capture-based methods like ViroCap enrich the pool of nucleic acid for viral DNA and RNA prior to sequencing, and allows investigation of the eukaryotic virome in greater detail [23]. Importantly, because the probes in ViroCap tile the complete genomes of the targets, we are subsequently able to carry out comparative analysis of the enriched, sequenced genomes. Our results are in agreement with previous reports regarding the differences in virome composition between laboratory, pet store $[29,30]$ and wild mice [31,32] and confirm that while most murine viral pathogens have been eradicated from lab mouse production facilities, these agents are abundant in non-laboratory populations [33].

The virome in mammalian hosts includes prokaryotic viruses (bacteriophages) that infect resident bacteria, eukaryotic viruses which transiently infect the host cells, and viral elements including retroviruses that are integrated into the host genome [34]. There is increasing evidence of a relationship between the eukaryotic virome and susceptibility to immune-mediated diseases such as inflammatory bowel disease (IBD) [35] and rheumatoid arthritis [36,37]. Furthermore, studies on the interaction between viruses and bacteria suggest direct inter-kingdom communication, and synergistic influences on the development of host immunity and susceptibility to various conditions [38]. The eukaryotic virome as a key component of the virome likely plays a critical role in host health and disease, including unidentified, subclinical viruses which may influence host physiology, immune system development, and disease/model susceptibility. As a consequence, there are potential influences on preclinical research investigating disease mechanisms, and development of novel therapeutics. All of these issues highlight the importance of a deeper understanding of the eukaryotic virome of mouse models [39,40].

To optimize mouse models of disease, a better understanding of the role of the microbiome, including the virome, in model phenotypes is needed. However, the variability in endogenous retroviruses remain. This finding was not unexpected given recent studies by Lee et al. [41] that used a TREome probe from murine leukemia virus-type endogenous retroviruses to survey C57BL/6J mice. They noted marked variability in the MLV-ERV landscape that depended on several factors, including individual mouse, sex, tissue, and cell type. What remains to be determined is the impact of such variation on individual mice as well as mouse models of disease in general.

Our study also identified murine astrovirus in both laboratory and pet store mice. Murine astrovirus was first found in nude mice in 1985, followed by the complete genome sequence from a wild mouse in 2011 [42,43]. The first complete murine astrovirus genome sequence that obtained from immunocompetent lab mice and published in 2012 [44]. Subsequent reports have confirmed the existence of astrovirus in laboratory mice [44,45], but the true prevalence in most research colonies remains unknown as it is not on many health monitoring profiles.

It has been speculated that the high prevalence of murine astrovirus in lab mice coupled with the diversity of virus strain [45] and asymptomatic infection could contribute to phenotypic differences between mice used in research.

This study also identified a murine norovirus in pet store that shared $92 \%$ nucleotide sequence identity to the next most-closely related strain. Murine noroviruses have been proposed as model agents for the study of human noroviruses [46,47]. However, unlike their human counterparts, which are a leading cause of non-bacterial epidemic gastroenteritis, murine noroviruses are asymptomatic unless infections occur in mice lacking anti-viral defense mechanisms [48]. However, their study has revealed novel putative roles for these viruses in intestinal homeostasis. For example, germ-free mice infected with MNV have increased the numbers of $\mathrm{CD} 4^{+}, \mathrm{CD} 8^{+} \mathrm{T}$ cells and IFN- $\gamma$ when compared to norovirus-free mice [49]. To this end, MNV-CR6 infection suppresses the expansion of group 2 innate lymphoid cells, a function similar to that of commensal bacteria [50]. In addition, MNV-CR6 infection of antibiotic-treated mice protected against DSS-induced intestinal injury. These findings suggest that noroviruses may play a physiological beneficial role in intestinal homeostasis. The identification of additional noroviruses such as that identified in this 
study provides further tools to understand the complex role of this family of viruses in health and disease.

\section{Conclusions}

Characterization of the microbiome of lab mice, pet store mice and wild mice stands to greatly aid our understanding of the crucial roles the microbiome play in host physiology and disease. Moreover, ensuring that the murine microbiome is representative of that seen in humans can yield more informative and translational mouse models of disease. Critical to this characterization and refinement is inclusion of the virome in discussions of the microbiome. Because lab mice are relatively free of viral pathogens, inclusion of studies of pet store or wild mice is needed to better incorporate the role of viruses. Collectively, such studies will also enhance our understanding of inter-kingdom interactions between viral and bacterial communities and the host.

Author Contributions: C.L.F. and A.C.E. conceived and designed the study. M.B. did some mouse work and performed DNA and RNA extraction. K.W. provided guidance for sequencing process. K.W. and B.H. participated the sequencing process. All authors were responsible for the collection and analysis of data. C.Z. prepared the manuscript. All authors read and revised the manuscript. All authors have read and agreed to the published version of the manuscript.

Funding: These studies were partly funded by the University of Missouri Mutant Mouse Resource and Research Center (MMRRC; NIH U42 OD010918).

Institutional Review Board Statement: The study was conducted according to the Guide for the Care and Use of Laboratory Animals and was approved by the University of Missouri Institutional Animal Care and Use Committee (protocol number 9587 and date of approval: 2016-2017).

Informed Consent Statement: Not applicable.

Data Availability Statement: The data supporting reported results can be found in this manuscript.

Acknowledgments: These studies were partly funded by the University of Missouri Mutant Mouse Resource and Research Center (MMRRC; NIH U42 OD010918). We would also like to acknowledge the animal care staffs at Discovery Ridge, the MU DNA Core and Informatics Research Core facilities, and the Comparative Metagenomics Laboratory.

Conflicts of Interest: The authors declare no conflict of interest.

\section{References}

1. Justice, M.J.; Dhillon, P. Using the mouse to model human disease: Increasing validity and reproducibility. Dis. Model. Mech. 2016, 9, 101-103. [CrossRef] [PubMed]

2. Vandamme, T.F. Use of rodents as models of human diseases. J. Pharm. Bioallied Sci. 2014, 6, 2-9. [CrossRef] [PubMed]

3. Perrin, S. Preclinical research: Make mouse studies work. Nature 2014, 507, 423-425. [CrossRef] [PubMed]

4. Collins, F.S.; Tabak, L.A. Policy: NIH plans to enhance reproducibility. Nature 2014, 505, 612-613. [CrossRef] [PubMed]

5. Wegorzewska, I.; Bell, S.; Cairns, N.J.; Miller, T.M.; Baloh, R.H. TDP-43 mutant transgenic mice develop features of ALS and frontotemporal lobar degeneration. Proc. Natl. Acad. Sci. USA 2009, 106, 18809-18814. [CrossRef] [PubMed]

6. Gill, A.; Kidd, J.; Vieira, F.; Thompson, K.; Perrin, S. No benefit from chronic lithium dosing in a sibling-matched, gender balanced, investigator-blinded trial using a standard mouse model of familial ALS. PLoS ONE 2009, 4, e6489. [CrossRef]

7. Pizzasegola, C.; Caron, I.; Daleno, C.; Ronchi, A.; Minoia, C.; Carri, M.T.; Bendotti, C. Treatment with lithium carbonate does not improve disease progression in two different strains of SOD1 mutant mice. Amyotroph. Lateral Scler. 2009, 10, 221-228. [CrossRef]

8. Seok, J.; Warren, H.S.; Cuenca, A.G.; Mindrinos, M.N.; Baker, H.V.; Xu, W.; Richards, D.R.; McDonald-Smith, G.P.; Gao, H.; Hennessy, L.; et al. Genomic responses in mouse models poorly mimic human inflammatory diseases. Proc. Natl. Acad. Sci. USA 2013, 110, 3507-3512. [CrossRef]

9. Visconti, A.; Le Roy, C.I.; Rosa, F.; Rossi, N.; Martin, T.C.; Mohney, R.P.; Li, W.; de Rinaldis, E.; Bell, J.T.; Venter, J.C.; et al. Interplay between the human gut microbiome and host metabolism. Nat. Commun. 2019, 10, 4505. [CrossRef]

10. Paone, P.; Cani, P.D. Mucus barrier, mucins and gut microbiota: The expected slimy partners? Gut 2020, 69, 2232-2243. [CrossRef]

11. Tlaskalova-Hogenova, H.; Stepankova, R.; Kozakova, H.; Hudcovic, T.; Vannucci, L.; Tuckova, L.; Rossmann, P.; Hrncir, T.; Kverka, M.; Zakostelska, Z.; et al. The role of gut microbiota (commensal bacteria) and the mucosal barrier in the pathogenesis of inflammatory and autoimmune diseases and cancer: Contribution of germ-free and gnotobiotic animal models of human diseases. Cell Mol. Immunol. 2011, 8, 110-120. [CrossRef] [PubMed] 
12. Shreiner, A.B.; Kao, J.Y.; Young, V.B. The gut microbiome in health and in disease. Curr. Opin. Gastroenterol. $2015,31,69-75$. [CrossRef] [PubMed]

13. Kamada, N.; Nunez, G. Regulation of the immune system by the resident intestinal bacteria. Gastroenterology 2014, 146, 1477-1488. [CrossRef] [PubMed]

14. Quigley, E.M. Gut bacteria in health and disease. Gastroenterol. Hepatol. 2013, 9, 560-569.

15. Kostic, A.D.; Chun, E.; Robertson, L.; Glickman, J.N.; Gallini, C.A.; Michaud, M.; Clancy, T.E.; Chung, D.C.; Lochhead, P.; Hold, G.L.; et al. Fusobacterium nucleatum potentiates intestinal tumorigenesis and modulates the tumor-immune microenvironment. Cell Host Microbe 2013, 14, 207-215. [CrossRef] [PubMed]

16. Le Chatelier, E.; Nielsen, T.; Qin, J.; Prifti, E.; Hildebrand, F.; Falony, G.; Almeida, M.; Arumugam, M.; Batto, J.M.; Kennedy, S.; et al. Richness of human gut microbiome correlates with metabolic markers. Nature 2013, 500, 541-546. [CrossRef]

17. de Vos, W.M.; de Vos, E.A. Role of the intestinal microbiome in health and disease: From correlation to causation. Nutr. Rev. 2012, 70 (Suppl. 1), S45-S56. [CrossRef]

18. Virgin, H.W. The virome in mammalian physiology and disease. Cell 2014, 157, 142-150. [CrossRef]

19. Karlsson, O.E.; Larsson, J.; Hayer, J.; Berg, M.; Jacobson, M. The Intestinal Eukaryotic Virome in Healthy and Diarrhoeic Neonatal Piglets. PLoS ONE 2016, 11, e0151481. [CrossRef]

20. Ericsson, A.C.; Davis, J.W.; Spollen, W.; Bivens, N.; Givan, S.; Hagan, C.E.; McIntosh, M.; Franklin, C.L. Effects of vendor and genetic background on the composition of the fecal microbiota of inbred mice. PLoS ONE 2015, 10, e0116704. [CrossRef]

21. Ericsson, A.C.; Montonye, D.R.; Smith, C.R.; Franklin, C.L. Modeling a Superorganism-Considerations Regarding the Use of "Dirty" Mice in Biomedical Research. Yale J. Biol. Med. 2017, 90, 361-371.

22. Zhang, C.; Franklin, C.L.; Ericsson, A.C. Consideration of Gut Microbiome in Murine Models of Diseases. Microorganisms 2021, 9, 1062. [CrossRef]

23. Wylie, T.N.; Wylie, K.M.; Herter, B.N.; Storch, G.A. Enhanced virome sequencing using targeted sequence capture. Genome Res. 2015, 25, 1910-1920. [CrossRef] [PubMed]

24. Peng, Y.; Leung, H.C.; Yiu, S.M.; Chin, F.Y. IDBA-UD: A de novo assembler for single-cell and metagenomic sequencing data with highly uneven depth. Bioinformatics 2012, 28, 1420-1428. [CrossRef] [PubMed]

25. Milne, I.; Stephen, G.; Bayer, M.; Cock, P.J.; Pritchard, L.; Cardle, L.; Shaw, P.D.; Marshall, D. Using Tablet for visual exploration of second-generation sequencing data. Brief. Bioinform. 2013, 14, 193-202. [CrossRef] [PubMed]

26. Li, H. A statistical framework for SNP calling, mutation discovery, association mapping and population genetical parameter estimation from sequencing data. Bioinformatics 2011, 27, 2987-2993. [CrossRef]

27. Briese, T.; Kapoor, A.; Mishra, N.; Jain, K.; Kumar, A.; Jabado, O.J.; Lipkin, W.I. Virome Capture Sequencing Enables Sensitive Viral Diagnosis and Comprehensive Virome Analysis. MBio 2015, 6, e01491-15. [CrossRef]

28. Prussin, A.J., 2nd; Marr, L.C.; Bibby, K.J. Challenges of studying viral aerosol metagenomics and communities in comparison with bacterial and fungal aerosols. FEMS Microbiol. Lett. 2014, 357, 1-9. [CrossRef]

29. Beura, L.K.; Hamilton, S.E.; Bi, K.; Schenkel, J.M.; Odumade, O.A.; Casey, K.A.; Thompson, E.A.; Fraser, K.A.; Rosato, P.C.; Filali-Mouhim, A.; et al. Normalizing the environment recapitulates adult human immune traits in laboratory mice. Nature 2016, 532, 512-516. [CrossRef]

30. Dammann, P.; Hilken, G.; Hueber, B.; Kohl, W.; Bappert, M.T.; Mahler, M. Infectious microorganisms in mice (Mus musculus) purchased from commercial pet shops in Germany. Lab. Anim. 2011, 45, 271-275. [CrossRef]

31. Rosshart, S.P.; Vassallo, B.G.; Angeletti, D.; Hutchinson, D.S.; Morgan, A.P.; Takeda, K.; Hickman, H.D.; McCulloch, J.A.; Badger, J.H.; Ajami, N.J.; et al. Wild Mouse Gut Microbiota Promotes Host Fitness and Improves Disease Resistance. Cell 2017, 171, 1015-1028.e1013. [CrossRef]

32. Farkas, T.; Fey, B.; Keller, G.; Martella, V.; Egyed, L. Molecular detection of novel astroviruses in wild and laboratory mice. Virus Genes 2012, 45, 518-525. [CrossRef]

33. Tao, L.; Reese, T.A. Making Mouse Models That Reflect Human Immune Responses. Trends Immunol. 2017, 38, 181-193. [CrossRef] [PubMed]

34. Maynard, C.L. 14-The Microbiota in Immunity and Inflammation. In Clinical Immunology, 5th ed.; Rich, R.R., Fleisher, T.A., Shearer, W.T., Schroeder, H.W., Frew, A.J., Weyand, C.M., Eds.; Content Repository Only: London, UK, 2019 ; pp. 207-219.e201.

35. Ungaro, F.; Massimino, L.; Furfaro, F.; Rimoldi, V.; Peyrin-Biroulet, L.; D'Alessio, S.; Danese, S. Metagenomic analysis of intestinal mucosa revealed a specific eukaryotic gut virome signature in early-diagnosed inflammatory bowel disease. Gut Microbes 2018, 10, 149-158. [CrossRef] [PubMed]

36. Raghavendra, P.; Pullaiah, T. Chapter 7-Pathogen Identification Using Novel Sequencing Methods. In Advances in Cell and Molecular Diagnostics; Raghavendra, P., Pullaiah, T., Eds.; Academic Press: Cambridge, MA, USA, 2018; pp. $161-202$.

37. Scher, J.U.; Sczesnak, A.; Longman, R.S.; Segata, N.; Ubeda, C.; Bielski, C.; Rostron, T.; Cerundolo, V.; Pamer, E.G.; Abramson, S.B.; et al. Expansion of intestinal Prevotella copri correlates with enhanced susceptibility to arthritis. Elife 2013, 2, e01202. [CrossRef]

38. Shi, Z.; Gewirtz, A.T. Together Forever: Bacterial-Viral Interactions in Infection and Immunity. Viruses 2018, 10, 122. [CrossRef] [PubMed]

39. Norman, J.M.; Handley, S.A.; Virgin, H.W. Kingdom-agnostic metagenomics and the importance of complete characterization of enteric microbial communities. Gastroenterology 2014, 146, 1459-1469. [CrossRef]

40. Wylie, K.M.; Weinstock, G.M.; Storch, G.A. Emerging view of the human virome. Transl. Res. 2012, 160, 283-290. [CrossRef] 
41. Lee, K.-H.; Lim, D.; Greenhalgh, D.; Cho, K. Highly Variable Genomic Landscape of Endogenous Retroviruses in the C57BL/6J Inbred Strain, Depending on Individual Mouse, Gender, Organ Type, and Organ Location. Int. J. Genom. 2017, 2017, 10. [CrossRef]

42. Phan, T.G.; Kapusinszky, B.; Wang, C.; Rose, R.K.; Lipton, H.L.; Delwart, E.L. The fecal viral flora of wild rodents. PLoS Pathog. 2011, 7, e1002218. [CrossRef]

43. Compton, S.R.; Booth, C.J.; Macy, J.D. Murine Astrovirus Infection and Transmission in Neonatal CD1 Mice. J. Am. Assoc. Lab. Anim. Sci. 2017, 56, 402-411.

44. Yokoyama, C.C.; Loh, J.; Zhao, G.; Stappenbeck, T.S.; Wang, D.; Huang, H.V.; Virgin, H.W.; Thackray, L.B. Adaptive immunity restricts replication of novel murine astroviruses. J. Virol. 2012, 86, 12262-12270. [CrossRef]

45. Ng, T.F.; Kondov, N.O.; Hayashimoto, N.; Uchida, R.; Cha, Y.; Beyer, A.I.; Wong, W.; Pesavento, P.A.; Suemizu, H.; Muench, M.O.; et al. Identification of an astrovirus commonly infecting laboratory mice in the US and Japan. PLoS ONE 2013, 8, e66937. [CrossRef]

46. Robilotti, E.; Deresinski, S.; Pinsky, B.A. Norovirus. Clin. Microbiol. Rev. 2015, 28, 134-164. [CrossRef] [PubMed]

47. Wobus, C.E.; Thackray, L.B.; Virgin, H.W.t. Murine norovirus: A model system to study norovirus biology and pathogenesis. J. Virol. 2006, 80, 5104-5112. [CrossRef] [PubMed]

48. Karst, S.M.; Wobus, C.E.; Lay, M.; Davidson, J.; Virgin, H.W. STAT1-dependent innate immunity to a Norwalk-like virus. Science 2003, 299, 1575-1578. [CrossRef]

49. Cadwell, K. Expanding the role of the virome: Commensalism in the gut. J. Virol. 2015, 89, 1951-1953. [CrossRef] [PubMed]

50. Kernbauer, E.; Ding, Y.; Cadwell, K. An enteric virus can replace the beneficial function of commensal bacteria. Nature 2014, 516, 94-98. [CrossRef] 